\title{
Best papers from the 12th Pacific-Asia conference on knowledge discovery and data mining (PAKDD2008)
}

\author{
Takashi Washio • Einoshin Suzuki • Kai Ming Ting
}

Published online: 12 October 2010

C Springer-Verlag London Limited 2010

The Pacific-Asia Conference on Knowledge Discovery and Data Mining (PAKDD) has been held every year since 1997. PAKDD 2008, the 12th in the series, was held in Osaka, Japan on May 20-23, 2008. PAKDD is a leading international conference in the area of data mining. It provides an international forum for researchers and industry practitioners to share their new ideas, original research results and practical development experiences from all KDD-related areas including data mining, data warehousing, machine learning, databases, statistics, knowledge acquisition, automatic scientific discovery, data visualization, causal induction and knowledge-based systems.

We received a total of 312 research papers from 34 countries and regions in Asia, Australia, North America, South America, Europe and Africa. Only approximately $11.9 \%$ of the these submissions were accepted as long papers, $12.8 \%$ of them were accepted as regular papers, and $11.5 \%$ of them were accepted as short papers upon rigorous reviews by two or three reviewers, discussions by the reviewers under the supervision of an area chair and judgment by the Program Committee Co-chairs. The highest rated papers in the review process were further evaluated by the Program Committee Co-chairs, and two papers were selected for the Best Paper Award and the Best Paper Runner-up Award. In addition to these award-winning papers, two outstanding papers were also nominated for this special issue. The authors of the four selected papers were asked to substantially extend the contents of their papers after the

T. Washio $(\bowtie)$

The Institute of Scientific and Industrial Research,

Osaka University, Ibaraki, Japan

e-mail:washio@ar.sanken.osaka-u.ac.jp

E. Suzuki

Graduate School of Information Science and Electrical Engineering,

Kyushu University, Fukuoka, Japan

e-mail: suzuki@i.kyushu-u.ac.jp

K. M. Ting

Gippsland School of Information Technology, Monash University, Melbourne, Australia

e-mail: kaiming.ting@infotech.monash.edu.au 
conference, and the extended papers were submitted for the review process of this special issue. After the review, only three out of the four papers were accepted for this special issue.

The first paper is entitled "A framework for modeling positive class expansion with single snapshot" and authored by Yang Yu and Zhi-Hua Zhou. It was awarded the best paper of PAKDD2008. This paper proposes a novel learning method of an accurate model from a single snapshot data and some limited domain knowledge under the scenario that the coverage of a target concept to be learnt is expanding over time. This work provides an entirely new aspect of learning in a dynamic environment from a single snapshot and static data.

The second paper is entitled "Multi-sorting algorithm for finding pairs of similar short substrings from large-scale string data" and authored by Takeaki Uno. It was awarded the runner-up best paper of PAKDD2008. This paper addresses the problem of finding pairs of strings with small Hamming distances from huge databases composed of short strings of a fixed length. It proposes an algorithm that runs in near-linear time in the input/output size. This near-linear algorithm is a breakthrough in the study of algorithms for finding similar substrings.

The third paper, entitled "Large-scale k-means clustering with user-centric privacypreservation" and authored by Jun Sakuma and Shigenobu Kobayashi, was selected for this special issue because of its high originality and significance enabling scalable privacypreserved k-means clustering among $n$ parties with $\mathrm{O}(\log n)$ time complexity. This paper proposes a k-means algorithm combined with a decentralized cryptographic protocol and a gossip-based protocol under unsynchronized participations of a large number of parties.

We wish to thank members of the PAKDD Steering Committee for their invaluable suggestions and supports throughout the organization process of the conference. We are indebted to the area chairs, Program Committee members and external reviewers of the conference in addition to the reviewers for this special issue for their effort and engagement in providing rich and rigorous comments to enhance the paper quality. We also want to thank the journal editor-in-chief Prof. Xindong Wu for his collaborative effort on this special issue. 